other cases with similar histories. What other diag. nosis could have been made than that of yellow fever. Anomalous dengue was one of the names suggested during the recent epidemic; if this is dengue, then the identity of that disease and yellow fever is established. When pressed for a diagnosis in certain of these cases, some of the Galveston experts denominated them Weil's disease, or acute infectious jaun dice, which is manifestly such a far fetched conclusion that words would be wasted in its refutation.

One of the arguments which has been repeatedly used against the existence of yellow fever in Texas during the past season, was the want of mortality. Upon this point let me quote Dr. Morris again $:^{3}$ "From Oct. 1, 1897 to Nov. 18, the death rate in Houston was $33 \frac{1}{3}$ per cent. greater than in the corresponding period of 1896." The causes given in death certificates are as follows: senility 11 (four of whom were not over 60 ); dengue 5; enteritis and gastritis 11 ; fever 10; meningitis 1; kidney disease 5. The record of Galveston does not show any increase of deaths during the months of August, September and October over the previous three years. There were five deaths however preceded by symptoms of yellow fever; it is a signicant circumstance that in four of these uremia was given in the certificate as causes of death. In the other, congestion of the lungs was mentioned as the primary, and bilious fever as the remote cause.

Those who deny the existence of yellow fever in Texas during the past season rely chiefly upon the apparent indisposition of the disease to spread from numerous foci. In other words, they contend that the presence of the infection necessarily involves its dissemination. No extended argument is requisite to demonstrate the fallacy of such conclusions. The pathogenic micro-organism of yellow fever is a facul. tative parasite, but its ordinary mode of life is sapro. phytic. It grows and develops outside of the body when the conditions are favorable for its reproduction. The seed may fall by the wayside, or upon a rock, or among thorns and wither away, or upon good ground and spring up and bear a hundred fold. 'The fact that the soil of Texas was an unfavorable one for the multiplication of the yellow fever germ last season is an adequate explanation of the history of the recent epidemic. It is not contended that the hygienic condition in these cities was perfect, but that the combination of circumstances was antagonistic to the extensive dispersion of the infection. Instead of being a blot upon the sanitary escutcheon of Houston and Galveston, it is another demonstration of the fact which has previously been observed, that yellow fever may be brought to these places without necessarily involving an extensive epidemic or serious mortality. Two important lessons should be emphasized; 1 , the urgency of promptly recognizing the early cases and calling them by their proper name, for by this means only may subsequent disaster in many instances be averted; 2, we should put our houses in order, in other words adopt every possible method of domiciliary and municipal cleanliness. Napoleon is said to have remarked that "Providence was on the side with the strongest artillery." When yellow fever is around Providence is on the side of the town with the best system of sewers. Again, it has been demonstrated that yellow fever may masquerade in the garb of dengue and that the latter is a portable disease; leaving out of the case the probable association with yel-

3 S. W. Med. Record, January, pp. 413-414. low fever, it became a serious question whether dengue should not be considered a quarantinable disease. The suffering and expense incident to an extensive epidemic like the one of last season would certainly appear to justify measures of prevention.

DISCUSSION.

Dr. Kinyoun of the U. S. Marine-Hosital Service-I have had some experience with yellow fever and dengue. Dr. West's paper is a very able one and he has gone into the points in differential diagnosis very thoroughly. I recall the history of the epidemic of dengue which occurred last year, April 12. The crucial test was made. In certain cities, not purposely but as a matter of convenience, persons were employed as disinfectore who had had dengue previously; none of these had the fever Catarrhal jaundice, dengue and mild yellow fever are unfortunately closely allied. And when we take this fact into con. sideration in the epidemic of last year it will be known that many cases of dengue were reported which were not dengue at all but mild cases of yellow fever.

Dr. BREwER of Texas-Jaundice occurs in about 50 per cent. of the cases. There were 600 cases and there were no deaths attributable to dengue fever. There was great excitement in the whole country but when it was found that there were no deaths following this epidemic, the excitement abated.

H. A. West of Galveston, Texas-I regret, Mr. Chairman, that this subject has not been discussed more fully, as the situation in Texas, imperfectly sketched in the paper, was of the most interesting a medical man is ever called upon to encounter. I would like very much to have heard the opinion of those whose experience has been wider than mine, especially as the narrated facts appear to controvert the generally accepted views as to the differential diagnosis of the fevers under consideration. The diagnosis of yellow fever in Galveston and Houston made by Dr. Guiteras was fought to a finish by the public prints, the people at large and by the majority of the physicians. It was iterated and reiterated that "yellow fever kills," "there is no such thing as mild yellow fever" and woe betide the man who had the temerity to raise his voice against the verity of this assumption. The actual mortality was hidden by certificates giving as the causes of death "senility, dengue, uremia, bilious fever, etc." The argument was sophistically presented by some as in the title of a paper upon the subject, "Dengue or Yellow Fever," that is to say it must be one thing or the other and leaving out of view entirely the probability of the concurrent prevalence of the two diseases. I have presented the facts, which are conclusive to my mind, that such was the case, also that yellow fever is not always and necessarily attended by a terrible mortality (this is one of the most instructive lessons of the recent epidemic, not only as to Texas, but in the regions east of the Mississippi); that it may not rapidly spread from numerous foci; that the symptomatol. ogy of yellow fever and dengue approaches more intimately than has hitherto been taught; and that in the light of the facts I have presented, the differential diagnosis of these fevers will have to be rewritten.

RARE CASES OF ARHYTHMIA.

Presented in the Section on Practice of Medicine at the Forty-ninth Annual Meeting of the American Medical Association, held at Denver, Colo., June 7-10, 1898.

BY JAMES M. ANDERS, M.D., PH.D., LL.D.

Professor of the Practice of Medicine and of Clinical Medicine in the Medico-Chirurgical College, Philadelphia: Attending Physician to the Medico-Chirurgical and Samaritan Hospitals, etc. PHILADELPHIA, PA.

Arhythmia is a symptom that is common to many diseases and conditions, but the scope of the present article is limited principally to the clinical features of certain forms of the disorder not hitherto described, that have fallen under my observation. The examples. that I shall adduce are too few to establish tenable hypotheses, either in their etiologic or clinico-pathologic bearings, yet they present points of rare clinical interest.

The first case occurred in the person of a physician, aged 40 years, who had been an abuser of tobacco for a protracted period, having habitually smoked from eight to twelve cigars daily. The family history gave undubitable evidence of a gouty tendency, and the 
patient himself had suffered from acute articular rheumatism on two different occasions. A careful physical examination permitted the positive exclusion of organic valvular diseases, and the probable elimination of other structural affections of the heart. Nor were any of the usual causative morbid conditions that usually precede or are found associated with arhythmia present, except the tobacco-habit.

The functional disturbance of the heart first assumed the form of true palpitation-i.e., the patient perceived the rapid cardiac pulsations, at the same time experiencing considerable mental anxiety and slight anginose pains. A little later a curious, and so far as I Rnow, peculiar form of arhythmia manifested itself. For example, on auscultation four sounds could be distinguished during cardiac rhythm, and it seemed clear that both the first and second sounds of the heart were reduplicated. The fact that the first sound was doubled was confirmed by the pulse, which was felt during the auscultation of the heart; the pulse-wave appeared to strike the palpating finger at the wrist synchronously with the second (feebler) element of the systole, while the impulse at the apex coincided with the first (stronger) element of the first sound. The pulse showed the modified double beat (pulsus bisferiens) when moderate pressure was made upon the radial. The pulsus bisferiens has been most frequently met with in combined aortic incompetency and aortic stenosis. The case here recorded is the only one (apart from a double aortic lesion) in my experience in which this form of pulse was typically shown. It is doubtless dependent upon double ventricular contraction. Elevation of both arms, so as to increase the force of the blood stream in the direction of the left ventricle, increased somewhat the irregularity, and accentuated the distinctive arhythmic features above described.

Broadbent" points out, also that "The pulsus bisferiens can not uncommonly be induced by an effort, which throws additional work upon the heart; for instance, in one case it was not present while the patient lay quietly in bed, but was brought out when he held up both his hands."

Cardiac action, in my case, was accelerated, the radial pulse ranging from 100 to 108 per minute. In a general way, the heart sounds resembled those of the well known canter rhythm, in which there is an interpolated sound, due in a majority of cases to the split. ting up of the second sound, but in the instance under consideration, a second interpolated sound was heard, following immediately upon the impulse, and it was ascribed, as before stated, to a double systole. To clearly show that the double second sound was a genuine reduplication in this case, I would direct especial attention to the following points: first, it was most distinctly heard at the base; secondly, the two elements of the duplicated sound were separated by an exceedingly brief interval, and finally, that both strongly resembled the normal second sound.

True reduplication of the second sound, which depends upon "a disturbance of the relation which normally exists between the pulmonary and aortic pressures," results from a great variety of causes. It is a well-established fact that in conditions in which the pulmonary tension is unduly high (mitral stenosis, etc., ) doubling of the second sound is common. Arthur G. Phear ${ }^{2}$ states that it may be met with in 1 Heart Diseases; With Special Reference to Prognosis and Treat. ment, p. 143. cases of anemia and debility, conditions that were not present in my cases. Phear has made an analysis of twenty-five cases of which detailed notes were available, with the following results: " $a$, mitral murmurs were present in ten cases, in five of which the murmur was presystolic only, in four systolic only, and in one double; $b$, in twelve cases, exclusive of the ten with mitral murmurs, anemia or debility was present, the individuals in many instances being convalescent from some acute febrile disease; $c$, two cases of acute lobar pneumonia, and $d$, in one case there was hypertrophy of the heart, with high tension pulse, and "bruit de galop' audible at the apex.",

I have carefully excluded so-called simulated reduplication, which is audible at the apex only, while at the base a single second sound is heard. Simulated reduplication, a rare condition, can not for lack of time and space be further considered here. A sphygmogram could not be conveniently obtained.

The patient was strongly advised to discontinue the smoking habit, and at his next visit, one month later, the peculiar form of arhythmia, to which the term "double reduplication" of the cardias sounds might be appropriately applied, had practically disappeared. Since then mild attacks of palpitation at long intervals, particularly as is usual on assuming recumbent posture at night and an occasional intermittences have been experienced.

A second instance of so-called "double reduplication "was observed in a female, under my charge in the Medico-Chirurgical Hospital, aged 43 , single, suffering from exophthalmic goiter in the advanced stage. The latter affection followed prolonged physical and mental strain. All of the characteristic symptoms had apparently developed in their usual sequence, i.e., tachycardia, muscular tremor, exophthalmos, and lastly, thyroidean enlargement. The cardiac impulse at the time of my first examination was greatly exaggerated; percussion dulness increased slightly, both to the right and the left, and auscultation rendered audible a blowing murmur over the base of the heart, while the valvular sounds were accentuated.

There was probably slight cardiac dilatation (secondary to functional hypertrophy) present, a common incident in this disease. The case immediately prior to admission (February 28) had, as stated before, reached an advanced period, with constant and markedly irregular action of the heart (delirium cordis). Twenty-four hours before death a gallop rhythm was clearly distinguishable on auscultation, while twelve hours previous to death, or at my last examination of the case, both the first and second sounds were apparently reduplicated. In this instance, also, the pulse was felt at the same time that auscultation was practiced. The pulsus bisferiens was not present. The redulplication in this instance, as is usual, was most clearly heard when the stethoscope was placed partly over the left and partly over the right ventricle near to the apex-beat. It was obviously caused by asynchronous contraction of the two ventricles.

A third case of arhythmia, while manifesting distinctly peculiar and rare features, was associated, as commonly occurs, with marked obesity. M. W., aged 58 years, widow, childless, weight 280 pounds, complexion ruddy, consulted me August 10, 1896, on account of inordinate dyspnea on slight exertion, and gastric disturbance. The family history was negative, except-

2 Lancet, January 9, 1897. 3 Loc. cit. 
ing the presence of positive evidence of a slight gouty taint. The patient had begun to take on flesh in a slow and gradual manner twenty years previously, though actual lipomatosis had not existed for more than ten years.

Condition at the time of examination.-The pulse was found to be wholly irregular, both in volume and rhythm, and increased in frequency to an average rate of 96 while the patient was at rest. On auscultating the heart while the pulse was felt at the wrist, it was noted that systole occasionally failed to send a pulsewave to the radial that the palpating finger could detect. Upon careful and repeated physical examination, which although far from satisfactory in its practical results on account of the enormous deposits of fat, valvular affections of the heart were not demonstrable. On the other hand, apart from the arhythmia noted before, nothing but the evidences of marked obesity and a feeble, muffled cardiac first sound were present.

As stated above, the condition at the time I first examined the case took the form of "combined arhythmia," $i$. e., irregularity in both volume and rhythm. .Shortly after, a typical "pulsus alternans" was occasionally noted. Recently, while the patient was suffering from an attack of influenza, I observed the "pulsus alternans" along with a modification of the rhythm that suggested an analogy to the CheyneStokes type of breathing. For example, after a comparatively short pause, or an intermittence, both the pulse and heart beat would begin at a slow rate and gradually increase in frequency for from eight to ten beats, at which moment the heart action would be quite rapid, to be followed by a second, abrupt pause, and so on.

There was present at the time the signs of a dry form of bronchitis (cough and sibilant râles on aus. cultation ), with slow, asthmatic breathing superadded. The periods of exacerbation or increasing acceleration of the cardiac contractions coincided, in the main, with the latter part of the inspiratory element of the respirations, while the pause following was synchronous with expiration. The remarkable influence of the respiratory function upon the cardiac action was further shown by the fact that during the held breath the heart rhythm at times became almost regular, at others it merely manifested decided improvement. I had previously witnessed, occasional intermittence and the "pulsus alternans" in morbus adiposis, but not the peculiar form of pulse or cyclical increase in the rate noted in this instance. Kisch ${ }^{4}$ contends that the "twin pulse" and obesity are conditions frequently found in association.

In the not infrequent irregularity of the cardiac action met with in the obese, an affection of which this case is a well marked example, I am inclined to believe that the condition is in great part dependent upon mechanical interference with the movements of the heart. That this embarrassment, however, is not wholly attributable to the depositions of fat, but partly to the respirations acting upon a heart already enfeebled by the toxins of influenza, is shown by the effect of a suspension of the latter function in the case reported above. Nothnagel ${ }^{5}$ has emphasized the fact that in meningitis the pulse may vary from minute to minute, the slow pulse, as in my own case, may

4 Berliner Klinische Wochengchrift, Berlin, March 18, 1895 .

5 Deutsche Archiv für Klinische Medicin in 1876, quoted by T. A. Claytor, University Medical Magazine, January, 1898. suddenly increase greatly in rapidity, and again become retarded. Baumgarten ${ }^{6}$ holds that these sudden oscillations are dependent solely upon central irritation. In the case reported here, however, the rise and fall of the pulse occurred almost synchronously with the respiratory acts, the variations or cycles occurred, therefore, from ten to twelve times in a minute. Again, it is a not infrequent matter of observation to find the presence of murmurs and various forms of arhythmia whose existence is totally dependent upon the influence of the respirations. In this connection, I may state that. I have observed cases in which apical systolic murmurs were audible only during the latter part of inspiration and the beginning of expiration. In one of these the murmur could be maintained for a considerable period during held inspiration. In another case, an occasional intermittence was also noted on inspiration. Neither of these two cases presented any evidences of organic or structural changes of either the heart or lungs; they both occurred, however, in neurasthenic subjects. The significance of the forms of arhythmia that I have described can not be positively stated, but they are probably grave in cases in which the cause or causes are not readily removable, as for example in the case of exophthalmic goiter reported above that proved speedily fatal.

In conclusion, I desire to restate in sequence some of the most interesting points embodied in this paper.

1. The presence of reduplication of both first and second sounds (double reduplication) in the first two cases, one of tobacco poisoning, the other exophthalmic goiter.

2. The occurrence of a typical pulsus bisferiens in case one, dependent upon the toxic effect of tobacco, in the absence of combined aortic regurgitation and stenosis.

3. The presence of a cyclical increase in the rapidity of the movements of the heart, followed regularly by a brief pause; and the striking effect mechanically of the respiratory function upon this peculiar variety of arhythmia.

4. The temporary production of apical systolic murmurs during the inspiratory element of the respiration.

5. That all of the cases developed in subjects in whom previously existing valvular lesions of the heart, so far as determined, were absent.

\section{THE PRESENT STATUS OF VACCINE AND VACCINATION.}

Presented to the Section on Practice of Medicine at the Forty-ninth Annual Meeting of the American Medical Association, held at Denver, Colo., June 7-10, 1897. BY W. F. ELGIN, M.D.,

ASSISTANT HYGIVNIC LABORATORY V. S. MARINE HOSPITAL SERVICE (A BUREAU OF THE TREASURY DEPARTMENT).

It will not be attempted in this article to go into a historical résumé of vaccination, but it may be profitable to discuss briefly the relation existing between smallpox and vaccinia. Since the beginning of this century, numerous experiments have been made to determine whether they are distinct diseases with interchangeable immunity, or whether vaccinia is an attenuated smallpox obtained by its passage through an animal of greater resistance. Gassner in 1801, Sunderland in 1828, Thiele in 1836, Ceely about the 6 Transactions of the Association of American Physicians, Vol. 1ii, 1888. 